\title{
The trouble with the Local Bubble
}

\author{
Barry Y. Welsh • Robin L. Shelton
}

Received: 18 March 2009 / Accepted: 29 May 2009 / Published online: 17 June 2009

(C) The Author(s) 2009. This article is published with open access at Springerlink.com

\begin{abstract}
The model of a Local Hot Bubble has been widely accepted as providing a framework that can explain the ubiquitous presence of the soft X-ray background diffuse emission. We summarize the current knowledge on this local interstellar region, paying particular reference to observations that sample emission from the presumed local million degree $\mathrm{K}$ hot plasma. However, we have listed numerous observations that are seemingly in conflict with the concept of a hot Local Bubble. In particular, the discovery of solar wind charge exchange that can generate an appreciable soft X-ray background signal within the heliosphere, has led to a reassessment of the generally accepted model that requires a hot local plasma.

In order to explain the majority of observations of the local plasma, we forward two new speculative models that describe the physical state of the local interstellar gas. One possible scenario is similar to the present widely accepted model of the Local Hot Bubble, except that it accounts for only $50 \%$ of the soft X-ray emission currently detected in the galactic plane, has a lower thermal pressure than previously thought, and its hot plasma is not as hot as previously believed. Although such a model can solve several difficulties with the traditional hot Local Bubble model, a heating mechanism for the dimmer and cooler gas remains to be found. The second possible explanation is that of the 'Hot Top' model, in which the Local Cavity is an old su-
\end{abstract}

B.Y. Welsh $(\bowtie)$

Space Sciences Laboratory, University of California, 7 Gauss

Way, Berkeley, CA 94720, USA

e-mail: bwelsh@ssl.berkeley.edu

R.L. Shelton

Department of Physics and Astronomy, University of Georgia, Athens, GA 30602, USA pernova remnant in which no (or very little) million degree local plasma is presently required. Instead, the cavity is now thought to be filled with partially ionized cloudlets of temperature $\sim 7000 \mathrm{~K}$ that are surrounded by lower density envelopes of photo-ionized gas of temperature $\sim 20,000 \mathrm{~K}$. Although this new scenario provides a natural explanation for many of the observations that were in conflict with the Local Hot Bubble model, we cannot (as yet) provide a satisfactory explanation or the emission levels observed in the B and Be ultra-soft X-ray bands.

Keywords Galaxy: general $\cdot$ ISM

\section{Introduction}

The stellar winds and supernovae events that are associated with clusters of massive early-type stars have a profound effect on the surrounding interstellar medium (ISM), including the creation of large cavities. These cavities, which are often referred to as "interstellar bubbles", are typically $\sim 100 \mathrm{pc}$ in diameter and have low neutral gas densities of $n(\mathrm{H}) \sim 0.01 \mathrm{~cm}^{-3}$ (Weaver et al. 1977). They are ubiquitously present both within our own Milky Way galaxy (e.g. the Eridanus, Cygnus, and Loop I superbubbles) and within the Large and Small Magellanic Clouds (e.g. N44 and N19). It is therefore unsurprising to learn that the Sun finds itself located within such a rarefied interstellar cavity (Welsh et al. 1998; Sfeir et al. 1999; Lallement et al. 2003), which we shall subsequently refer to as the Local Cavity. The Sun's placement within a region of unusually low gas density has been known for over 40 years and was initially deduced from the very low values of interstellar extinction measured for stars within a distance of $\sim 100 \mathrm{pc}$, as compared with the reddening derived 
for the more distant regions of the galaxy (Fitzgerald 1968; Lucke 1978). In addition, the small H I column densities derived from Ly-alpha absorption measurements of nearby stars (Bohlin 1975) and the lack of spectral hardening in the observed soft X-ray background signal, which requires a low value of local neutral gas absorption (Bowyer and Field 1968), also added credence to the belief that the solar neighborhood resides within a region of low neutral hydrogen density.

During the 1970's observations of a ubiquitous $0.25 \mathrm{keV}$ soft X-ray background signal by the Wisconsin group (Sanders et al. 1977; Williamson et al. 1974) and the detection of the O VI (1032) absorption line towards several OB stars located within the solar neighborhood by the Copernicus satellite (Jenkins 1978) provided strong supporting evidence that the Local Cavity was filled with a highly ionized and hot (million degree K), low density plasma. In addition, new theoretical models of the general ISM predicted the presence of a pervasive hot gas-phase component (Cox and Smith 1974; McKee and Ostriker 1978). Given that other superbubbles, such as those of Cygnus (Cash et al. 1980) and the Orion-Eridanus region (Reynolds and Ogden 1979), were also being detected through their X-ray emission properties and that Heiles (1979) had just compiled an extensive list of galactic bubbles/supershells observed in the radio $21 \mathrm{~cm}$ H Iline, the observational and theoretical basis for believing that hot gas resided in the cavity seemed well substantiated (Cox and Reynolds 1987). This hot interstellar plasma was dubbed the Local Hot Bubble.

At the time of the Local Bubble \& Beyond Conference (Breitschwerdt et al. 1998) the widely accepted picture was one in which the Sun is located within an extremely low neutral density $\left(n(\mathrm{H} \mathrm{I}) \sim 0.005 \mathrm{~cm}^{-3}\right)$, high thermal temperature $\left(T \sim 10^{6} \mathrm{~K}\right)$ and highly ionized local plasma with dimensions of at least $80 \mathrm{pc}$ in many galactic directions. This high temperature plasma produces half to all of the observed soft X-ray background intensity in most galactic directions. The hot gas seen in low latitude directions must be local because there are nearby optically thick walls of H I (the neutral boundary to the Local Cavity) towards which a non-zero $1 / 4 \mathrm{keV}$ flux is observed (Snowden et al. 2000). Lying within the rarefied and hot bubble are many low density $\left(N(\mathrm{H} \mathrm{I}) \sim 0.1 \mathrm{~cm}^{-3}\right)$, partially ionized $\left(n_{e} \sim 0.1 \mathrm{~cm}^{-3}\right)$ and warm $(T \sim 7000 \mathrm{~K})$ cloudlets, the most well-studied being the local interstellar cloud, within which the Sun is thought to reside (Lallement and Bertin 1992). The origin of the Local Hot Bubble was thought to be linked to an explosive event in the nearby $(d \sim 150 \mathrm{pc})$ ScoCen OB association that occurred some $2 \mathrm{Myr}$ ago (Frisch 1981), or one or more nearby SN (Cox and Anderson 1982; Edgar and Cox 1993), such that the local region can essentially be considered as an old cooling SNR or superbubble structure.
The observed anticorrelation of the soft X-ray background emission intensity with the total sight-line neutral hydrogen column density led to two main models that attempted to explain the observed spatial distribution of the $\mathrm{X}$-ray emitting gas. In the absorption model the ubiquitous soft X-ray background emission was thought to originate outside the Galaxy and to be partially absorbed by Galactic material, although the absorption cross sections could not produce the observed dynamic ranges (McCammon et al. 1976) or band ratios (Bloch et al. 1986; Juda and Bloch 1991). In the widely accepted displacement model of the Local Hot Bubble (Sanders et al. 1977; Cox and Reynolds 1987), the observed soft X-ray background brightness was thought to be proportional to the path length through the hot Local Bubble gas, which is roughly anticorrelated with the total hydrogen gas column density. Although neither model could fully explain all of the observations of the soft X-ray background, the displacement model was stronger. The prevailing thought was that the soft $\mathrm{X}$-ray background emission signal observed along any sight-line is composed of 4 components: (i) emission from the Local Hot Bubble of interstellar plasma located in the galactic disk near to the Sun, (ii) emission from a more distant (halo) source of soft $\mathrm{X}$-rays located at high galactic latitudes, (iii) emission from an isotropic extragalactic background and (iv) an emission component arising in the Galactic disk at distances beyond the absorbing wall of the Local Cavity (Kuntz and Snowden 2000).

Such a model could account for the relative scarcity of neutral gas and dust for interstellar sight-lines that extend $<100 \mathrm{pc}$ (Frisch and York 1983). Furthermore, cold and dense high latitude clouds could block emission originating from a more distant halo and/or extragalactic origin, thus creating observable 'shadows' in the maps of the diffuse soft X-ray background emission (Burrows and Mendenhall 1991; Snowden et al. 1993). By analyzing these shadows, it has been possible to isolate the contribution of foreground Local Bubble emission from more distant emission. These shadowing experiments have provided the strongest evidence for a million degree plasma located within a hot Local Bubble, with a typical contribution of $\sim 400 \times 10^{-6} \mathrm{R} 12$ (i.e. ROSAT R1 and R2 band) counts s $\mathrm{s}^{-1} \operatorname{arcmin}^{-2}$ to the total $1 / 4 \mathrm{keV}$ soft $\mathrm{X}$-ray background signal in most directions (Snowden et al. 2000).

We discuss additional properties of the hot local gas in Sect. 2. Several discrepancies between the model of the Local Hot Bubble and the observations are also discussed in this section. The most difficult problem is that posed by the discovery of solar wind X-rays, which appear to account for a significant fraction of the flux that had been attributed to a million degree local plasma. Taking into account the solar wind X-ray contribution, we outline possible revisions to the Local Hot Bubble model in Sects. 3 and 4. Section 3 describes the least drastic revision, that in which the emission 


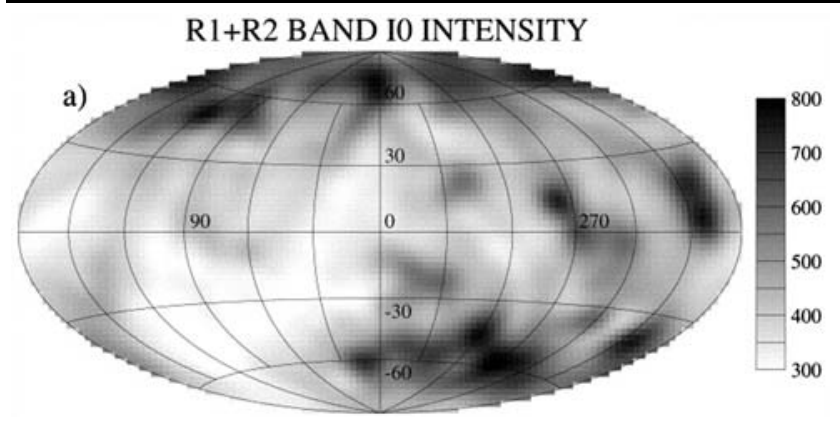

Fig. 1 The galactic distribution of the summed R1 (0.11-0.284 keV) and R2 $(0.14-0.284 \mathrm{keV})$ band emission as recorded by the ROSAT all-sky survey (Snowden et al. 1998). Note that the highest intensities are generally found at high galactic latitudes

from a hot local plasma is simply dimmer in soft X-rays than previously believed. Section 4 describes the other extreme, a new model called the Hot Top Model, in which the solar wind X-rays account for all of the local X-rays in the Galactic plane.

\section{Details of, and problems with, the Local Hot Bubble model}

In the following subsections we briefly discuss each of the major physical properties of the standard Local Hot Bubble model (i.e. temperature, pressure, size, etc). Over the past decade observational evidence has emerged that has raised serious questions concerning the standard model of a hot local plasma. Therefore, we also make note of observations that would seem to contradict several of the shibboleths concerning how the local ISM is currently perceived.

\subsection{The spectrum of the $10^{6} \mathrm{~K}$ plasma}

Diffuse emission arising from a seemingly ubiquitous $0.25 \mathrm{keV}$ soft X-ray background (soft X-ray background) signal has been widely observed in the $\mathrm{Be}, \mathrm{B}$, and $\mathrm{C}$ bands during numerous suborbital sounding rocket flights by the (McCammon and Sanders 1990; McCammon et al. 2002) and during the ROSAT soft X-ray background all-sky survey (Snowden et al. 1998). The galactic distribution of this emission, as recorded by this latter survey, is shown in Fig. 1.

In the Galactic plane, apart from directions towards SNRs, superbubbles, or the Galactic center, observations record only the emission that originates within the Local Hot Bubble. X-ray shadowing observations using high latitude clouds have also isolated this hot and local component. The observed ratio of flux in the $\mathrm{B}$ band relative to that in the $\mathrm{C}$ band and the ratio of flux in the ROSAT R1 band relative to that in the ROSAT R2 band are indicators of the plasma's temperature and are roughly constant across the sky. The prevailing explanation for these observations is that the $\mathrm{X}$-ray emission arises in a pool of hot gas $\left(T \sim 10^{6} \mathrm{~K}\right)$ which surrounds the Sun and whose radius varies with direction but whose properties do not. There is a 2-sigma upper limit on the intermixed $\mathrm{H}$ I column density within the Local Hot Bubble of $N(\mathrm{H} \mathrm{I})<6.6 \times 10^{18} \mathrm{~cm}^{-2}$ (Juda and Bloch 1991). This limit is in agreement with the inferred average $\mathrm{H}$ I column density throughout the Local Cavity as derived from $\mathrm{Na}$ I observations along sight-lines within $60 \mathrm{pc}$ (Welsh et al. 1994). However, as discussed in Sect. 2.4, the shape of the Local Hot Bubble, which is calculated from the assumption of uniform emissivity, is difficult to reconcile with the shape of the surrounding structure. The ROSAT and Wisconsin X-ray broad-band data are generally consistent with collisional ionizational equilibrium (CIE) emission models for a gas at 1 million ${ }^{m o} \mathrm{~K}$. Using higher resolution spectra of the hot emitting gas the Chandra, XMM-Newton, and Suzaku satellites have also found that the data in the 0.4 to $0.7 \mathrm{keV}$ band can be fit with CIE model spectra (Markevitch et al. 2003; Galleazzi et al. 2007; Henley et al. 2007). The actual spectrum of the soft X-ray background in this energy range is seen to be dominated by emission from the O VII and O VIII lines. However, CIE models that generate the observed surface brightness of the $\mathrm{O}$ VII line are found to predict too much lower energy $1 / 4 \mathrm{keV}$ band emission for the MBM 12 direction (Smith et al. 2007).

In a few cases theoretical models in which the X-ray emitting gas was slightly out of equilibrium such that the plasma is slightly underionized could not be disallowed (Smith et al. 2005; Henley et al. 2007). We note that models having a highly non-equilibrium ionization (NEI) condition in which the plasma is thought to be strongly over-ionized due to a previous event such as a supernova (Breitschwerdt and Schmutzler 1999; Breitschwerdt 2001) are seemingly disallowed by several observations. Recent FUSE observations of the emission intensity of both O VI (Shelton 2003) and C III (Welsh et al. 2002) cannot be reconciled with the NEI model of the Local Hot Bubble (Breitschwerdt 2001) which implies line intensities far greater than those which are observed.

Additionally, observations of the diffuse extreme ultraviolet (EUV) sky background spectral emission using both the CHIPS (Hurwitz et al. 2005) and EURD (Edelstein et al. 2001) satellites are also in contradiction with the predictions of the NEI model. We also note that strong Si III (1206 ̊) interstellar absorption has been reported for several sight-lines towards nearby stars (Holberg et al. 1999; Nehme et al. 2008). This ion has a relatively high rate coefficient for charge exchange with neutral hydrogen and its presence within the local interstellar medium is unlikely to be linked to the remnant of some past ionization event such as a supernova shock (Lyu and Bruhweiler 1996; 
Fig. 2 The distribution of cold and neutral gas within $\pm 250 \mathrm{pc}$ of the Sun in the galactic plane as revealed by interstellar $\mathrm{Na} \mathrm{I}$ absorption measurements (Lallement et al. 2003). Dark contour regions represent neutral gas with $\log N(\mathrm{H} \mathrm{I})>$ $19.3 \mathrm{~cm}^{-2}$; white regions are of low gas density typically of $\log N(\mathrm{H} \mathrm{I})<18.3 \mathrm{~cm}^{-2}$. This is a view looking down onto the galaxy with the Sun at the center of the plot. Note the narrow extensions of the low density Local Cavity into surrounding interstellar cavities such as the Beta CMa tunnel, the Pleiades and Loop I bubbles

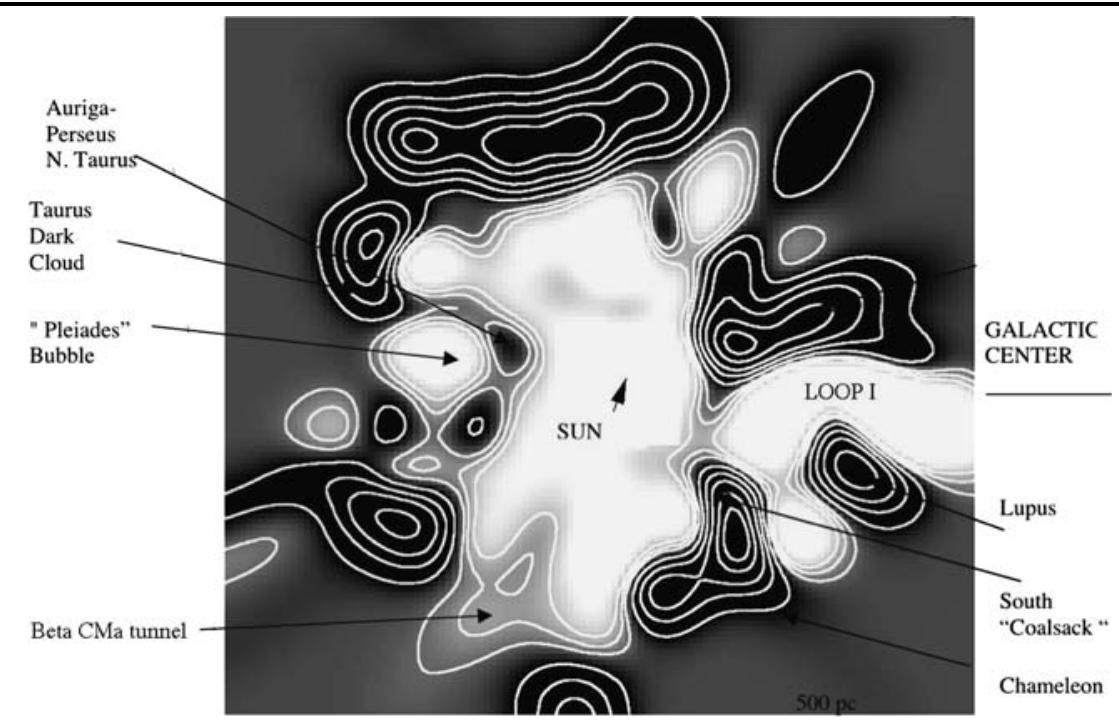

Breitschwerdt and Schmutzler 1999). Depending on the details of the local ionizing radiation field, a high hydrogen ionization fraction $(95 \%)$ is required in order to sustain the presently observed amounts of Si III.

Two sets of high spectral resolution observations of the soft X-ray background have been made thus far (Sanders et al. 2001; McCammon et al. 2002). The 148 to $284 \mathrm{eV}$ observations by Sanders et al. found a blend of emission lines, whose spectrum cannot be reproduced by a solar abundance CIE plasma. This energy region is the most sensitive to the effects of absorption and the observed region was in the galactic plane, therefore the emission must originate in a local source of hot gas. We note that a major difficulty with the CIE emission model is that a million degree local plasma should also be bright at extreme ultraviolet (EUV) wavelengths, but attempts to detect the associated EUV line emission have not been successful (Jelinsky et al. 1995, 1998; Hurwitz et al. 2005). Although adherents to a Local Hot Bubble can explain these non-detections as being due to the local hot gas having a very low gas-phase iron abundance (thus making the detection of the expectedly strong Fe IX line at $171 \AA$ problematic), we note that Fe is unlikely to be depleted in typical hot ISM plasma (Yao et al. 2006).

\subsection{Cool gas inside and outside the local hot bubble}

The bubble of local hot gas is assumed to fit within the Local Cavity, whose location is defined by the "walls" of cold and neutral material surrounding the local region. The size and shape of the Local Cavity is best estimated from high spectral resolution optical studies of the $\mathrm{Na}$ I D-lines (5890) seen in absorption towards many sight-lines within $300 \mathrm{pc}$. The $\mathrm{Na} \mathrm{I}$ ion is a good tracer of cold $(T<1000 \mathrm{~K})$ and neutral $(I . P .<5.1 \mathrm{eV})$ interstellar gas and has been widely used as a surrogate for local H I absorption (Ferlet et al. 1985;

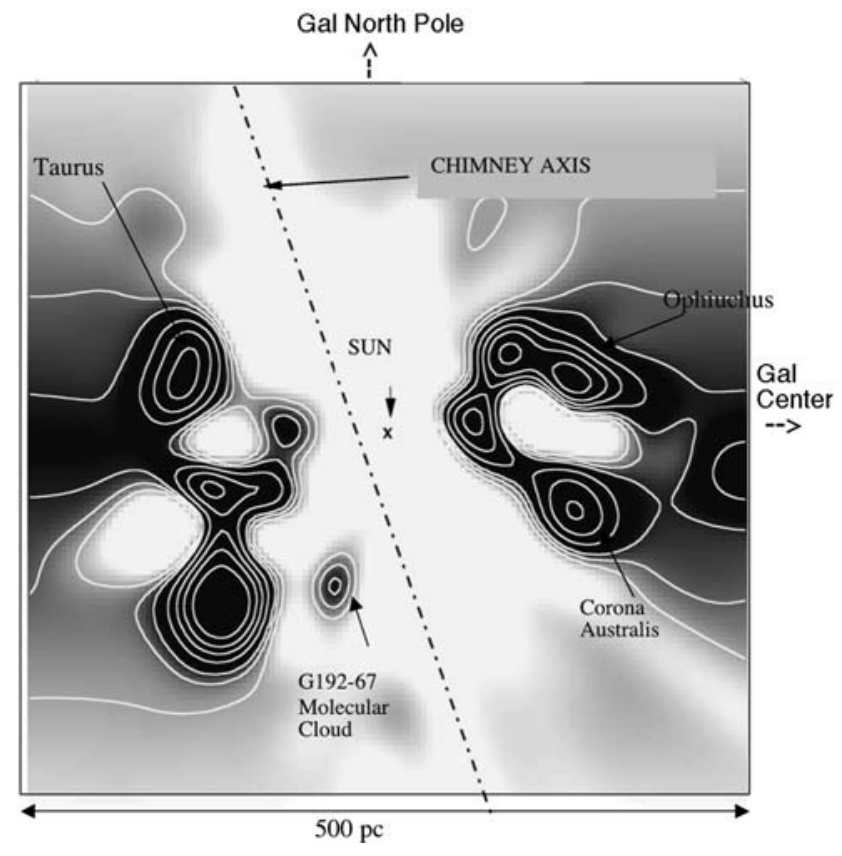

Fig. 3 The distribution of cold and neutral gas within $\pm 250 \mathrm{pc}$ of the Sun in the plane perpendicular to the galactic plane as revealed by interstellar Na I absorption measurements (Lallement et al. 2003). Dark contour regions represent neutral gas with $\log N(\mathrm{H} \mathrm{I})>$ $19.3 \mathrm{~cm}^{-2}$; white regions are of low gas density typically of $\log N(\mathrm{H} \mathrm{I})$ $<18.3 \mathrm{~cm}^{-2}$. This is a view looking side-on to the galaxy with the Sun at the center of the plot. Note the extension of the Local Cavity into the overlying halo via the low density Local Chimney feature, which is tilted at an angle that points towards the regions of highest soft X-ray background emission

Welsh et al. 1994). In a recent $\mathrm{Na}$ I absorption survey of 1005 sight-lines within 250 pc (Lallement et al. 2003), 3-D maps of the density distribution of cold gas have revealed the Local Cavity to be an irregularly shaped rarefied region of 50-80 pc radius in most directions in the Galactic plane. 
Figure 2 shows that the Local Cavity is connected to adjacent low density interstellar regions (such as the Pleiades bubble and Loop III) through narrow interstellar tunnels, as predicted by theoretical models of the ISM (Cox and Smith 1974). These maps of the distribution of cold and neutral local gas reveal 3 interesting interstellar features: (i) a $200 \mathrm{pc}$ long by $50 \mathrm{pc}$ wide extension of the Local Cavity towards the direction of the star Beta CMa, (ii) extensions of the Local Cavity into the lower galactic halo through both of the open-ends of a rarefied Local Chimney feature (Welsh et al. 1999), see Fig. 3 and (iii) a connection through a disturbed interstellar region that opens into the Loop I superbubble at a distance of $\sim 90 \mathrm{pc}$ (Welsh and Lallement 2005). We shall return later to the significance these latter two features in Sect. 3 .

UV and optical absorption measurements towards stellar targets within $100 \mathrm{pc}$ of the Sun have revealed the presence of many partially ionized and warm diffuse cloudlets located within the Local Cavity (Lallement et al. 2003; Redfield and Linsky 2008). These cloudlets typically have hydrogen column densities, $\log N(\mathrm{H} \mathrm{I}) \sim 10^{17}-10^{18} \mathrm{~cm}^{-2}$ and temperatures of $<7000 \mathrm{~K}$ and possess bulk motions generally differing by only $5-10 \mathrm{~km} \mathrm{~s}^{-1}$. Their presence within the Local Hot Bubble is thought to provide natural sites for transition temperature layers where ions such as O VI could reside (Slavin and Frisch 2002). The most well-studied of these cloudlets is the local interstellar cloud, partially within which the Sun is thought to reside. The cloudlets within the Local Cavity should provide very weak absorption sites for the more distant soft X-ray emission, thus not interfering with the X-ray spectroscopic and shadowing studies discussed previously. However, only a very few cold $(T<1000 \mathrm{~K})$ and dense gas clouds and no molecular clouds, which can more efficiently absorb soft X-rays, are known to exist within the Local Cavity (Lallement et al. 2003).

\subsection{Transition temperature gas: O VI}

One of the earliest pieces of observational evidence for a hot and highly ionized local plasma came from the detection of the $\mathrm{O}$ VI ion seen in the UV absorption spectra of nearby $(d<150 \mathrm{pc}$ ) B-type stars by the Copernicus satellite (Jenkins and Meloy 1974; Jenkins 1978). These findings have been substantiated by the recent FUSE surveys of O VI absorption detected towards hot white dwarf stars within 200 pc of the Sun (Oegerle et al. 2005; Savage and Lehner 2006). We note that O VI (1032 $\AA$ ) is a tracer of transition-temperature gas at $\sim 300,000 \mathrm{~K}$, and because of its quick cooling rate, theoretical production models require a source of hotter gas for its formation and continual replenishement. The $\mathrm{O}$ VI could exist in a transition zone between the hot million K Local Hot Bubble plasma and cooler gas clouds. The prevailing interpretation of these data is that the $\mathrm{O}$ VI resides on the periphery of the Local Cavity and/or on evaporating interfaces located between the (numerous) partially ionized cloudlets which are embedded within the Local Hot Bubble. The FUSE O VI detections were only made on local sight-lines with distances $>35 \mathrm{pc}$ and with typical values of column density of $\log N(\mathrm{O} \mathrm{VI}) \sim 13.0 \mathrm{~cm}^{-2}$.

However, recent re-examinations of $\mathrm{O}$ VI data forward evidence that argues against the presence of this ion being located within the rarefied confines of the Local Hot Bubble, whose contours have been discussed in Sect. 2.2. Firstly, for the 5 B-stars with (Hipparcos) distances $<75$ pc (i.e. the nominal distance to the edge of the Local Cavity in most directions) the Copernicus data reveal only one (albeit tentative) detection of interstellar O VI absorption (Jenkins 1978; Bowen et al. 2008). We note that there is a large variation between the (one) measured O VI column density and the (four) upper limit values for these very local sight-lines, which is unlikely to be due to any $\mathrm{O}$ VI absorption that would exist with a million $\mathrm{K}$ plasma. Instead, this effect has been attributed to a variation in the character of the transition temperature zones surrounding individual clouds.

A new detailed analysis of the radial velocities of interstellar and (when present) stellar absorption lines in a sample of $\sim 100$ absorption spectra recorded by FUSE for hot white dwarfs within $400 \mathrm{pc}$ of the Sun has recently been made available (Barstow et al. 2008, 2009). For 2/3 of the sight-lines having significant $\mathrm{O}$ VI features the velocity of the $\mathrm{O}$ VI absorption is found to lie within the velocity uncertainty (i.e. $14 \mathrm{~km} \mathrm{~s}^{-1}$ ) of features that Barstow et al deemed to be photospheric in origin, and thus an interstellar origin cannot be confirmed. In most of the remaining cases the sight-lines with confirmed interstellar O VI absorption extend to or just beyond the neutral boundary of the Local Cavity and are at high galactic latitudes. Similar findings for the location of local O VI absorption, but at lower latitudes, have also been made using a small sample of B-type stars located $>80 \mathrm{pc}$ from the Sun (Welsh and Lallement 2008).

Since the local interstellar cloud is widely thought to be (at least partially) surrounded by a hot Local Bubble plasma, it could therefore be a prime evaporative transition region for the production of high ions. Several authors have therefore constructed theoretical models that provide predictions for the numbers of associated $\mathrm{O}$ VI ions, which if spread over most of the cloud's surface should be detected in the absorption spectra recorded along most more distant sight-lines (Slavin 1989; Borkowski et al. 1990; Slavin and Frisch 2002; Cox and Helenius 2003). Most models predict absorption column densities in the $\log N(\mathrm{O} \mathrm{VI})=12.5$ to $13.0 \mathrm{~cm}^{-2}$ range, which is marginally consistent with what is detected by the FUSE O VI surveys along local sight-lines. Since one might expect to encounter more than one conductive cloud interface over the $>40 \mathrm{pc}$ sight-lines in which 
FUSE has detected O VI, then the observations suggest that something may be reducing the effectiveness of thermal conduction in these O VI-producing interfaces. Jenkins (2008) has proposed that local clouds could mutually shield each other from the effects of a hot Local Bubble plasma, such that a conduction front suitable for the formation of $\mathrm{O}$ VI only occurs at the very periphery of the cloud grouping. Alternately, others have shown the importance of magnetic fields in their ability to inhibit conduction (Slavin 1989; Cox and Helenius 2003). Although the strength of the magnetic field in the case of the local interstellar cloud has been estimated to be of a low value of $\sim 2 \mu \mathrm{G}$ (Wood et al. 2007), if the magnetic field is tangled then this could account for the low values of $\mathrm{O} \mathrm{VI}$ ion production along certain sight-lines.

\subsection{Size and shape of the local hot bubble}

The approximate size and shape of the emitting region of the local hot plasma has been estimated under the assumption that the emissivity per volume is constant throughout the Local Hot Bubble (Snowden et al. 1990, 1998). This assumption implies that the Local Hot Bubble's surface brightness in any given direction is linearly proportional to its radius. The actual distance scaling was determined from observations aimed toward the shadowing molecular cloud MBM 12, which appeared to be placed within or just beyond the edge of the Local Hot Bubble. The initial calculations assumed a distance of $\sim 65 \mathrm{pc}$ to this molecular cloud. However, more recent estimates of the distance to MBM 12 now place it at a distance $>200$ pc (Luhman 2001; Chauvin et al. 2002), such that the main absorber of the more distant emission in this direction is instead provided by the neutral gas wall to the Local Cavity which is located at a distance of $\sim 100 \mathrm{pc}$. Thus, the revised radius of the hot bubble cavity should be about 1.5 times greater than the $50 \mathrm{pc}$ to $100 \mathrm{pc}$ range found previously.

Both the (previous and revised) estimated shape and size of the Local Hot Bubble derived from X-ray observations do not match the detailed contours of the Local Cavity derived by the Na I mapping method and shown in Figs. 2 and 3. If the Local Cavity is uniformly filled with hot emitting plasma then the significant extension of the cavity to a distance of $\sim 200 \mathrm{pc}$ in the direction of the star $\beta$ CMa would be expected to show an enhanced level of soft X-ray background emission, which is not seen in the data (Snowden et al. 1998). However, there is at least some concordance that the first $50 \mathrm{pc}$ in all directions it contains very little cold and dense neutral gas (although there are several partially ionized clouds within this volume). Furthermore, the ROSAT soft X-ray maps find that the Local Hot Bubble is unusually bright in two high latitude regions that are offset by $\sim 45^{\mathrm{mo}}$ from the north and south poles (Snowden et al. 1998). These directions have been found to be well aligned with the openings of the Local Chimney into the halo (Welsh et al. 1999), as shown in Fig. 3. Additionally, it is well-known that there is a dipole effect in the distribution the soft X-ray background emission at mid-plane latitudes, with the emission being warmer, of greater intensity and with a larger spatial coverage in directions towards the galactic center than sightlines towards the galactic anti-center (Snowden et al. 2000). The present model of the Local Hot Bubble provides no substantial explanation for this effect. However, this effect can be explained through inspection of the $\mathrm{Na}$ I density map shown in Fig. 2, which clearly shows that there are many more gaps in the neutral boundary of the Local Cavity at mid-plane galactic latitudes between $190^{\circ}<l<330^{\circ}$ (that could potentially allow the passage of more distant generated soft X-rays) than in the opposite galactic direction.

Finally, we note that an alternative view has been forwarded in which the Local Hot Bubble is smaller than the Local Cavity, leaving a space in the interstellar medium between the inner hot gas and the outer dense gas of the cavity walls. The properties of the material within this space are unknown, and how such a situation could have arisen or be sustained has not been adequately explained.

\subsection{Thermal pressure of the hot gas}

The derived pressure of the local hot gas has been a longstanding puzzle, together with the global problem of the apparent pressure imbalance between galactic disk gas and that of the overlying galactic halo (Cox and Reynolds 1987). Supporting the halo requires a gas pressure of $P / k=7,000$ to $10,000 \mathrm{~cm}^{-3} \mathrm{~K}$ (Shelton et al. 2007), suggesting that the Local Hot Bubble should have a similar pressure. More recent local pressure estimates of $P / k \sim 15,000 \mathrm{~cm}^{-3} \mathrm{~K}$ have been derived from soft X-ray and EUV shadowing observations towards several nearby cold interstellar clouds (Snowden et al. 1993; Kuntz et al. 1997; Burrows and Guo 1998; Berghofer et al. 1998). In contrast, measurements of thermal pressures of several diffuse gas clouds residing inside the Local Hot Bubble result in far lower pressure values (Jenkins 2002). There clearly seems to be a pressure imbalance between the hot and colder gas within the LISM. In particular such an imbalance would tend to crush the cool clouds residing within the Local Hot Bubble and also then to try and expand the size of Local Cavity, neither of these effects has been observed.

However, this apparent local pressure anomaly could be accounted for in that (i) the previous distance estimates to the cold clouds used in these shadow experiments have now been revised (see Lallement et al 2003) such that a lower value of $P / k$ for the hot gas pressure is now obtained (i.e. $P / k \sim 12,250 \mathrm{~cm}^{-3} \mathrm{~K}$ due to the revision of the clear pathlength in the MBM 12 direction from $65 \mathrm{pc}$ to $100 \mathrm{pc}$, the distance to the Local Cavity boundary), and/or (ii) any remaining pressure difference could be accounted for by interstellar magnetic and/or turbulent gas pressure. However, 
even with conservative revisions to these data, the spectre of a pressure imbalance still remains. A more thorough discussion of the issues that arise from this apparent pressure imbalance can be found in Jenkins (2008).

\subsection{Kinematics of the local cavity gas}

Although the main physical properties of the local hot gas have been determined from soft X-ray background observations, the limited spectral resolution of these emissionline data preclude a detailed kinematic assessment of gas velocity. However the motions of lower temperature gas residing within the Local Cavity can be derived from the absorption profiles of UV and optical lines observed at high spectral resolution along sight-lines towards nearby stars (Redfield and Linsky 2008; Savage and Lehner 2006; Crawford et al. 1998).

For the case of the numerous partially ionized gas clouds of temperature $\sim 10^{4} \mathrm{~K}$ that are known to reside within $\sim 50 \mathrm{pc}$ of the Sun, their velocity vectors are roughly parallel with a general flow originating in the Scorpio-Centaurus association. The velocity amplitudes encompass a wide range of values between clouds, suggesting that interstellar shocks may be prevalent. The turbulent velocity in some of these clouds is high, suggesting the presence or recent existence of shocks, which could result in the return of metals to the gas phase through the shock dissipation of dust grains (Redfield and Linsky 2008).

For higher temperature $\left(T \sim 10^{5} \mathrm{~K}\right)$ gas located within the Local Cavity, most of the local O VI absorption velocities correlate well with the lower temperature ion velocities (Savage and Lehner 2006). This is to be expected if O VI is formed in a condensing interface between cool and hot Local Cavity gas. There are also some sight-lines in which the $\mathrm{O}$ VI absorption velocity is displaced to positive velocities from the lower temperature gas, which suggests in these cases that the $\mathrm{O}$ VI may be tracing an evaporative flow from interface regions.

Measurements of the absorption velocity of the Na I neutral gas associated with the nominal boundary to the Local Cavity do not suggest that the Local Cavity wall is expanding or contracting with any significant gross motions (Lallement, private communication). This would be expected if the Local Cavity is an old SNR whose expansion has been significantly slowed down by its interaction with the surrounding dense ISM. Also, if the Local Cavity is collapsing (such as being due to a marked pressure imbalance) then it would collapse at the sound speed, which is not observed.

Finally we note that there are two major dynamical movements of gas inflow into the Local Cavity region. The first, as mentioned earlier, is a flow of gas clouds into the Local Cavity from the adjacent Loop I superbubble (i.e. the ScoCen association). This inflow has been traced using both low (Ca II, S II, Fe II) and high ionization (C IV, Si IV, $\mathrm{N}$ V) species, all of which possess significant negative velocity motions for sight-lines of distance $>90 \mathrm{pc}$ in the general direction of $l=330^{\circ}, b=+18^{\circ}$ (Welsh and Lallement 2005). Secondly, for galactic directions close to the axis of the Local Chimney that extend into the lower halo, interstellar gas clouds with inflow velocities in the -20 to $-60 \mathrm{~km} / \mathrm{s}$ range have been detected along sight-lines spanning both open-ends of the Local Chimney which appear to be falling towards the galactic disk (Welsh et al. 2004; Crawford et al. 2002).

\subsection{Origin and age of the local hot bubble and the local cavity}

The history and age of the Local Hot Bubble are much debated. It is widely believed that the Local Hot Bubble and Local Cavity are the result of one or more supernova (SN) explosions (Cox and Anderson 1982), but in an alternate scenario this superbubble picture is disputed, and it is instead the rarefied Local Cavity that is thought to be caused by star formation epochs in the Scorpius-Centaurus OB association as regulated by the nearby spiral arm configuration (Bochkarev 1987; Frisch 1995; Fuchs et al. 2006; Maiz-Apellaniz 2001). Other suggested Local Cavity formation scenarios involve an association with the young and massive stars of the Gould Belt (Grenier 2000) or the passage of a high velocity cloud through the galactic disk (Comeron and Torra 1994). The present day morphology of the Local Cavity seems to be defined by the 'void' within the ISM created by the outer shells of nearby overlapping superbubble structures (Welsh et al. 1994; Frisch 1995).

Recent modeling of the joint evolution of the Loop I and Local Hot Bubble superbubbles involve $\sim 20 \mathrm{SNe}$ occurring in the moving group of $\mathrm{OB}$ stars of the Sco-Cen cluster which passed through the present day local cavity (Breitschwerdt and de Avillez 2006). In such a scenario the formation age of the Local Hot Bubble is constrained to $\sim 14.5 \mathrm{Myr}$, with the last re-heating occurring $\sim 0.5 \mathrm{Myr}$ ago. In a similar supernova driven formation model the last re-heating episode is placed at $\sim 3$ million years ago (Smith and Cox 2001), based on the excess abundance of ${ }^{60} \mathrm{Fe}$ found in the Earth's deep ocean crust (Fields et al. 2005).

\subsection{Solar wind charge exchange (SWCX)}

At the Local Bubble conference in 1997, Freyberg (1998) and Cox (1998) separately recognized that the then-unknown $\mathrm{X}$-ray production mechanism which made Comet Hyakutake surprisingly $\mathrm{X}$-ray bright may operate elsewhere in the solar system. They warned astronomers that these X-rays could pose a significant problem for interpretations of the 
soft X-ray emission from the Local Hot Bubble. It was soon realized that the source of such cometary X-rays was the deexcitation of solar wind ions following charge exchange with neutral material (Cravens 1997, 2000). Very highly ionized and fast moving solar wind ions have a large cross section for interacting with neutral material in cometary and planetary atmospheres, as well as neutral material that has been swept into the heliosphere. During the interaction, called solar wind charge exchange (sometimes referred to as SWCX), an electron from the neutral material is transferred into a highly excited state in the solar wind ion followed by a decay that produces one or more high energy X-ray photons (Wargelin et al 2008).

Coronal mass ejections, which enhance the solar wind density in localized regions for hours to days, are probably responsible for the long term enhancements seen in the ROSAT data and nominally removed from the ROSAT All Sky Survey (Snowden et al. 1997, 1998). The Sun's slow and fast winds, which are less sporadic but vary in relative contribution during the 12 year solar cycle, create a slowly varying component which has not been removed from the ROSAT All Sky Survey. Since solar wind charge exchange $\mathrm{X}$-rays are produced locally, they also behave as a local component in all shadowing observations. This signal level has generally been attributed to emission associated with the Local Hot Bubble. Lallement (2004), Cravens et al. (2001) and Robertson and Cravens (2003) have assessed the potential level of contamination to the ROSAT All Sky Survey by solar wind charge exchange in the slow and fast winds. Robertson and Cravens (2003) found that 0.1 to $1.0 \mathrm{keV} \mathrm{X}$-rays produced in the heliosphere by the steady slow and fast winds could account for $\sim 1 / 2$ of the soft X-rays in the Galactic plane and a smaller fraction of the high latitude locallymade soft X-rays. Similarly, Lallement (2004) calculated the steady, heliospheric solar wind charge exchange X-ray intensity in the $1 / 4 \mathrm{keV}$ band, the band in which the local $\mathrm{X}$-ray emission is most prominent, finding that solar wind charge exchange could explain most of the $1 / 4 \mathrm{keV}$ intensity that was previously attributed to emission from the Local Hot Bubble. Both sets of estimates are uncertain, due to uncertainties in the neutral ion density, solar wind density, interaction cross section, and solar wind charge exchange spectrum, making precise estimates difficult. Even when the upper limits are used in these global predictions, the level of predicted solar wind charge exchange X-ray emission cannot explain the excess of local X-rays observed at high latitudes. In addition, the maps of Robertson and Cravens (2003) show significant structure at low latitudes. The ROSAT maps do not show corresponding dim regions, suggesting that the Local Hot Bubble's X-ray emission has compensated for inhomogeneities in the solar wind charge exchanges. However, an alternate conclusion is that the solar wind charge exchange $\mathrm{X}$-rays are more evenly distributed than in the Robertson \& Cravens maps.
Koutroumpa et al. (2006, 2007, 2008a) have made more detailed calculations of the solar wind charge exchange spectrum, finding it to be somewhat harder than that attributable to the nominal Local Hot Bubble. As a result, they conclude that solar wind charge exchange can explain all of the local $3 / 4 \mathrm{keV}$ emission found from the ROSAT All Sky Survey and all of the local O VII $570 \mathrm{eV}$ and $\mathrm{O}$ VIII $650 \mathrm{eV}$ line emission seen in pointed observations by Chandra, XMM-Newton, and Suzaku. More recent calculations by Koutroumpa et al. (2009) that compare the calculated solar wind charge exchange spectra with soft X-ray shadow fields observed with ROSAT reveal that the solar wind charge exchange intensity is approximately independent of latitude. The intensity of the solar wind charge exchange emission in the ROSAT $1 / 4 \mathrm{keV}$ band is, on average, $\sim 332 \times 10^{-6}$ counts s$^{-1}$ arcmin $^{-2}$, which is of the same order as the soft $\mathrm{X}$-ray background intensity that was previously attributed to emission from the Local Hot Bubble at low galactic latitudes. However in high latitude regions $\left(b> \pm 40^{\circ}\right)$ the calculated levels of solar wind charge exchange emission cannot explain the high levels of $1 / 4 \mathrm{keV}$ emission observed by ROSAT and thus a hot emitting gas in the galactic halo is required.

The calculations of Koutroumpa et al suggest that the solar wind charge exchange spectrum may be harder than previously thought. This notion agrees with the observations of the soft X-ray background spectrum (Sanders et al. 2001; McCammon et al. 2002). However, we note that these latter data were collected from a large area of the sky centered close to the axis of the Local Chimney at high northern galactic latitudes and thus presumably sampled significant amounts of (distant) emission from the hot and ionized million degree $\mathrm{K}$ halo. Both sets of the soft X-ray background measurements cannot explain all of the observed $1 / 4 \mathrm{keV}$ emission and even less of the B-band emission recorded by the Wisconsin surveys, in addition to being in conflict with the EUV spectral data. Thus, the Local Hot Bubble spectrum is required to be softer then previously thought, and the soft X-ray emission shortfall could be filled by a warm ( $T<10^{6} \mathrm{~K}$ ) emitting gas within the Local Cavity.

\section{Ramifications for the 'accepted local hot bubble model'}

It is clear from the preceding sections that several important problems plague the traditional model of the Local Hot Bubble. The most important of these is the contamination by solar wind charge exchange $\mathrm{X}$-rays. The predicted severity of the $1 / 4$ and $3 / 4 \mathrm{keV} \mathrm{X}$-ray contamination by this heliospherically generated emission for low latitude sightlines ranges from $\sim 1 / 2$ in the model of Robertson and Cravens (2003) to approximately $100 \%$ from the calculations of Koutroumpa et al. (2009). Thus, new models of the 
local region range from those that are $\sim 1 / 2$ as bright in $\mathrm{X}$ rays as the traditional Local Hot Bubble models to those that have no local hot gas in the Galactic plane. In either case, the model must include $\mathrm{X}$-ray producing gas located at high latitudes because solar wind charge exchange models cannot explain all of the observed high latitude X-ray emission. In this subsection, we consider the first case in which the Local Hot Bubble is half as bright in the plane as previously believed. In the following section, we consider the other extreme, that in which gas in the local ISM produces none of the soft X-rays seen at low latitudes.

Firstly, if emission from a Local Hot Bubble is diminished relative to traditional models, it is diminished at all latitudes by a similar amount $\left(\sim 330 \times 10^{-6}\right.$ counts s $^{-1} \operatorname{arcsec}^{-2}$ in the ROSAT 1/4 keV band) according to Koutroumpa et al. (2008a). Subtracting a constant intensity from the observed anisotropic distribution of locally produced X-rays leaves a more extremely anisotropic distribution of X-rays which can then be attributed to the Local Hot Bubble. If we make the standard assumption that the X-ray emissivity is constant throughout this emitting region, then the Local Hot Bubble must be extremely distorted, with strong lobes in the northern and southern hemispheres and a tight waist in the Galactic plane. Secondly, the model solar wind charge exchange spectra are harder than the observed spectrum. We conclude this because the solar wind charge exchange predictions of Koutroumpa et al. account for a greater fraction of the observed $\mathrm{X}$-rays in the $1 / 4 \mathrm{keV}$ band than in the Wisconsin $\mathrm{B}$ band (130 to $188 \mathrm{eV}$ ). In order to compensate for the hardness of the solar wind charge exchange spectrum, the Local Hot Bubble spectrum must be softer than previously believed. Thus, the local emitting plasma would need to be cooler than previously believed.

Any revisions to the X-ray brightness and temperature require that the electron density be recalculated. If the temperature and path length are unaffected, then a reduction in the Local Hot Bubble emission intensity by a factor of 2 would imply a reduction in the electron density by a factor of $1 / \sqrt{2}$, resulting in $n_{e} \sim 0.005 \mathrm{~cm}^{-3}$. This serves as a reasonable starting point. However, once the solar wind charge exchange contamination is better understood, this estimate can be improved upon by an improved derivation of the temperature of the Local Hot Bubble plasma (and thus the emissivity) and the intensity of Local Hot Bubble X-rays in the direction of MBM12. Because the electron density and temperature are less than previously believed, the thermal pressure must also be less. The reduction in the electron density by $1 / \sqrt{2}$ alone, brings the estimated thermal pressure down from $12,250 \mathrm{~K} \mathrm{~cm}^{-3}$ to $\sim 8,700 \mathrm{~K} \mathrm{~cm}^{-3}$. Any revision to the local plasma temperature will lower the gas pressure further.

These revisions reduce or resolve some of the problems posed by observations. Firstly, it may now be pos- sible for the hot gas in the Local Cavity to be in pressure balance with the embedded warm clouds, especially if the expected downward revisions to the Local Hot Bubble's thermal pressure are severe and if the clouds are somewhat supported by non-thermal pressures such as magnetic pressure and turbulence. Furthermore, the reduction in the Local Hot Bubble X-ray intensity may help to explain the low fluxes of the Fe IX, Fe X, and Fe XI photons observed by in ultra-soft X-ray spectra (McCammon et al. 2002; Hurwitz et al. 2005). The precise estimates of the expected Fe IX, Fe X, and Fe XI intensities, however, depend on the Local Hot Bubble's (newer lower) temperature, which is not yet known.

These revisions also re-open existing inquiries. The heating mechanism for the hot gas must be re-evaluated now that the gas is found to be dimmer and cooler, and the spatial distribution of the hot gas must also be reconsidered. The previously estimated shape of the emitting cavity, which was determined from the $1 / 4 \mathrm{keV} \mathrm{X}$-ray intensity and the assumption that the intensity scaled with the pathlength, disagreed with the shape of the Local Cavity, but such disagreement may evaporate once the spatial distribution of Local Hot Bubble X-ray emission is better known. In addition, given that the bubble's X-ray intensity is less than previously thought, the source and history of the Local Hot Bubble must be reworked. A single or multiple supernova may have heated the gas, but such events were probably fewer and possibly happened further back in the past than currently believed.

As previously noted, some of the observed physical parameters of the LHB appear to be consistent with the characteristics of a fossil SNR. The Local Cavity is not expanding at the expense of its surroundings, just as fossil remnant cavities are no longer driven by the over-pressure of expanding gas within them. However, the hot gas once contained within the Local Cavity may have escaped through the Local Chimney into the galactic halo, or it may have cooled due to turbulent mixing or conduction with entrained gas or gas close to the galactic plane. The Local Cavity structure may now be gently photo-ablating in the residual UV field. Shelton (1998) has discussed the possibility that the Local Cavity may be an aging SNR. In her model, she notes that once a cool expansion shell forms any O VI should form within and at the periphery of the hot bubble, which is moving far slower than the SN shock front. Once the SNR shell forms, the soft X-ray emission (from the remnant hot gas) ceases to be edge-brightened and its emission diminishes by an order of magnitude. In addition, she finds that the $\mathrm{X}$-ray productivity of older SNRs depends critically on the ambient non-thermal pressure, thus allowing the possibility that an ancient SNR in a diffuse environment may possess a higher pressure than previously thought. Because such a 
model does not explain the observed local spatial distribution of O VI which appears to be mostly formed at the periphery of the cavity as opposed to being distributed within the cavity itself, we suggest that most of the observed O VI is due to the interface between the cavity gas and the hot halo gas. In addition, without an additional source of heating, old remnants tend to collapse such that the material around a fossil SNR will have moved back to fill in the low pressure space, and there will not be an observable cavity.

We now turn to a speculative model of the Local Cavity in which solar wind charge exchange explains all of the observed soft X-ray emission in the Galactic plane, i.e. the local interstellar medium is devoid of hot gas in the plane. In addition, the cavity is filled with warm $(20,000 \mathrm{~K})$ photoionized gas, some of which may have entered the aging cavity from the Sco-Cen bubble. Although such a model represents a radically different view, it goes a long way in resolving many of the outstanding issues that currently bedevil the generally accepted model of a Local Hot Bubble.

\section{The 'hot top model'}

The following is a possible scenario for the physical state of the Local Cavity. It is not based on a rigorous theoretical framework but is, instead, driven directly by recent observational evidence. Although it is far from complete, it attempts to accommodate the ramifications of the recent observations of solar wind charge exchange, O VI and the EUV background emission. Although referred to here as a 'model', it is really a descriptive framework designed to stimulate theorists into re-assessing the currently accepted models of a hot Local Bubble. In addition, it has testable predictions that future observations in the UV and/or soft X-ray regimes will be able to confirm or refute. Bearing these caveats in mind, we assume the following:

The size and shape of the Local Cavity is defined by the neutral contours derived from $\mathrm{Na}$ I absorption measurements (Lallement et al. 2003), such that the absorbing wall that surrounds most of the Local Cavity can be categorized by $\log N(\mathrm{H} \mathrm{I})>19.3 \mathrm{~cm}^{-2}$. A possible new picture of the Local Cavity is one in which it is mostly filled with a very diffuse and photoionized and/or collisionally ionized gas of temperature $\sim 20,000 \mathrm{~K}$ that is in rough pressure equilibrium $\left(P / k \sim 3000 \mathrm{~cm}^{-3}\right)$ with the numerous partially ionized and warm $(T \sim 7000 \mathrm{~K})$ small cloudlets that are known to exist within the LISM. The average electron density of the ionized Local Cavity 'filler gas' is assumed to be $n_{e} \sim 0.04 \mathrm{~cm}^{-3}$ (derived from pressure equilibrium with the partially ionized LISM cloudlets) and there is also a very low average neutral gas density of $n(\mathrm{H})<$ $0.01 \mathrm{~cm}^{-3}$ within the Local Cavity (Dupin and Gry 1998; Welsh et al. 1994). We note that there may also be a contribution from magnetic pressure such that the total pressure of both the local interstellar cloud and 'filler gas' may be higher than we have assumed. ${ }^{1}$

Our model also requires that the major source of local non-solar wind charge exchange generated soft X-rays occurs at high galactic latitudes $\left(b> \pm 35^{\circ}\right)$, which is probably due to emission from a hot and ionized gas viewed through the openings of the Local Chimney in to the overlying halo. Also there is a highly absorbed component generated in surrounding hot (X-ray emitting) superbubble cavities that abut the Local Cavity within the thick galactic disk. A simple schematic of the model is shown in Fig. 3.

Our assumption that the Local Cavity is mostly filled with warm and photoionized gas is based on observations of the Ar I, N II, C III and Si III ions that have been widely observed throughout the local ISM (Lehner et al. 2003) and by the recognition that the shortage of neutral argon in nearby clouds can be better explained by photoionization rather than collisional ionization (Sofia and Jenkins 1998). In addition, photo-ionization conditions are observed to vary considerably throughout the LISM (Lehner et al. 2003). For example, Wolff et al. (1999) have found evidence for an ionization gradient in the local gas with an ionization increase along the general direction to the Canis Major extension to the Local Cavity.

Most back-of-the-envelope calculations assume that the plasma is in collisional ionization equilibrium throughout the Local Cavity, although there is no real consensus on this matter and such models do not fit the observed soft X-ray background spectrum (Sanders et al. 2001; Breitschwerdt 2001). Bruhweiler and Cheng (1988) and Vallerga (1998) have taken a different approach by considering the photoionizing effects of nearby hot stars. Photoionization from the local EUV radiation field (which is dominated by the EUV flux from the two B stars $\epsilon$ and $\beta$ CMa, together with a few nearby hot white dwarfs) can account for the high interstellar gas electron density measured towards nearby stars, although the spectrum is too soft to explain the apparent overionization of helium with respect to hydrogen measured in the local cloud (Vallerga and Slavin 1998). Several authors have invoked additional (non-equilibrium) sources of ionization to explain this anomaly (Slavin and Frisch 2002). In one model, the shocks that resulted from a local supernova event that may have occurred $\sim 3.6$ million years ago are the main cause of the time-dependent ionization at the Sun, although most of the photons with wavelengths $>500 \AA$ are due to the stellar spectra (Lyu and Bruhweiler 1996). Thus, the supposed shock ionization event, from which the gas has yet to return to its equilibrium state, is supplemented by the

\footnotetext{
${ }^{1}$ Although we use the term 'filler' gas, in reality the photo-ionized regions will be the rarefied ionized envelopes surrounding cooler interstellar cloud cores. Since the Local Cavity has numerous cloudlets of this type, these outer cloud envelopes will probably merge into a photoionized medium that can best be described as a 'filler' gas.
} 
present stellar radiation field. The ionization rate for this is $\Gamma(\mathrm{H} \mathrm{I})=1.1 \times 10^{-15} \mathrm{~s}^{-1}$ (Vallerga and Welsh 1995). We note the long recombination time of hydrogen in the ISM, which for $10 \%$ ionization and $n(\mathrm{H} \mathrm{I}) \sim 0.1 \mathrm{~cm}^{-3}$ is about $10^{7}$ years (Chassefiere et al. 1986). The timespan since the supposed supernova explosion also agrees, roughly, with the data gained from the ferromanganese ocean layer crust of the Earth, for which a local supernova event is required to have happened 3 Myrs ago (Knie et al. 2004; Fields et al. 2005). Thus, although we have invoked photo(and some collisional) ionization in our new model, the long time-scales for $\mathrm{H}$ recombination and the current availability of sufficiently strong stellar photo-ionization sources seems adequate to sustain both the ionization and temperature of our theorized Local Cavity filler gas.

From the various solar wind charge exchange calculations of Koutroumpa et al., Lallement, and Robertson \& Cravens, we know that there may be far less X-ray emitting interstellar gas in the local region than previously expected. As noted in Sect. 2.8, simulations of the contribution of solar wind charge exchange spectra in ROSAT R1 and R2 observations of shadow field sight-lines by Koutroumpa et al. (2008a, 2009) indicate that the level of solar wind charge exchange flux is not strongly latitude dependent, but can account for almost all of the observed R1 and R2 emission at low galactic latitudes (i.e. within the Local Cavity). However, an unabsorbed $1 / 4 \mathrm{keV}$ soft X-ray background component is seen to be well correlated with the absolute galactic latitude for galactic latitudes of $b> \pm 35^{\circ}$. Thus, a high latitude interstellar component exists and the spatial distribution of its emission seems directionally correlated with sight-lines viewed through both openings of the Local Chimney into the lower galactic halo (Welsh et al. 1999).

We therefore conjecture that at high latitudes the observed soft X-ray background emission (which is thought to arise in a hot and highly ionized halo) is only weakly absorbed when viewed through the low H I column sight-lines that are contained within the 'openings' of the Local Chimney, and thus the soft X-ray background is detected with a greater intensity in these directions. For sight-lines into the halo that do not pass through these low opacity openings, the soft X-ray background emission signal is highly absorbed by the higher $\mathrm{H}$ I density gas in the galactic plane. This is in accord with the ROSAT shadow observations which argued against a single emission component from a hot halo of large scale-height (Snowden et al. 2000), but instead required a low scale-height variable (in both temperature and intensity) emission component, which could also be considered as the superposition of multiple, spatially distinct emitting components. Additionally, the ends of the Local Cavity near the Chimney openings may be filled with hot gas that has flowed into it from above.

We know that there is anomalous structure in the direction of the northern chimney opening because of polariza-

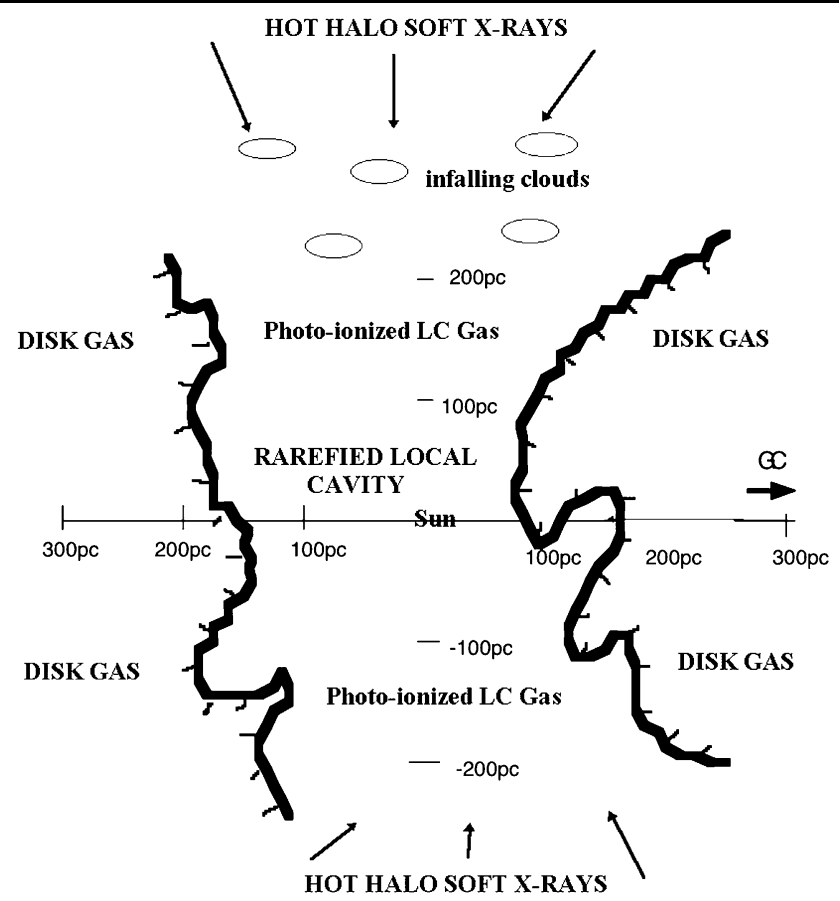

Fig. 4 Simple 3-D schematic of the 'Hot Top' Model. The figure shows the spatial distribution of low neutral density photo-ionized gas contained within the Local Cavity surrounded by its cold and neutral gas boundary wall and its openings into the halo through the Local Chimney as viewed in the meridian plane (Lallement et al. 2003). The entire region is subject to incident soft X-ray emission, arising in a million degree hot halo, that impinges on infalling gas clouds at high galactic latitudes

tion data. Mathewson and Ford (1970) have measured the polarization to nearby stars and found that for sight-lines $<50 \mathrm{pc}$ the E-vectors have low polarization values and are also well ordered with no apparent major disturbances in the local magnetic field, as also concluded by Tinbergen (1982). However, for sight-lines in the 50 to $100 \mathrm{pc}$ range there is a clear change in both the magnitude and direction of the Evectors which rise up from the galactic plane to high galactic latitudes spanning the galactic longitude range $\ell=300$ to $+30^{\circ}$. Additionally, using radio polarization data, Wolleben (2007) has formulated a new model for the Loop I radio feature that involves two X-ray emitting shells. The region of overlap of these two expanding shells of Loop I matches most of the disturbed region of E-vectors in the 50-100 pc range noted by Mathewson and Ford (1970). A similarly disturbed region is also predicted by Wolleben at the southern opening of the Local Chimney.

The idea for our new picture of the Local Cavity was largely driven by an interpretation of the results from a recent re-analysis of O VI absorption profiles detected by the FUSE satellite towards local hot white dwarfs (Barstow et al. 2008, 2009). Apart from a few instances, the new Barstow et al. results largely agree with and extend upon previous measurements which contained a smaller sample 


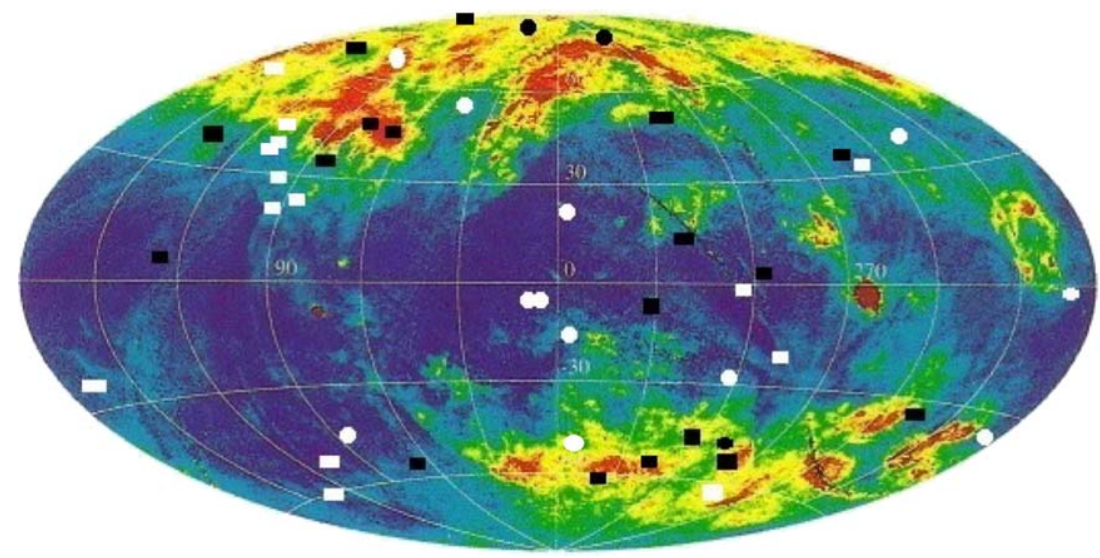

Fig. 5 Spatial distribution of local O VI absorption towards local hot white dwarfs (Barstow et al. 2009; Savage and Lehner 2006) and B-type stars within 120 pc (Bowen et al. 2008; Welsh and Lallement 2008) superposed on a map of the distribution of the soft X-ray background R12 emission (Snowden et al. 1998). Sight-lines with distances $<80 \mathrm{pc}$ are circles, sight-lines with distances $>80 \mathrm{pc}$ are squares. Filled black circles or squares are detections of O VI, whereas

of hot white dwarf targets whose spectra were reduced using an earlier version of the FUSE data processing pipeline (Oegerle et al. 2005; Savage and Lehner 2006). In Fig. 4 we show the spatial distribution of the $1 / 4 \mathrm{keV} \mathrm{R} 1$ and R2 soft X-ray background emission (Snowden et al. 1998) together with the galactic positions of local $(d<200 \mathrm{pc})$ hot white dwarfs that have undisputed detections (and nondetections) of interstellar O VI absorption (i.e. those sightlines in which the velocity difference between interstellar and photospheric O VI absorption is greater than the FUSE spectral resolution). In addition we have similarly plotted $\mathrm{O}$ VI data from the 4 sight-lines within $120 \mathrm{pc}$ (Savage and Lehner 2006) that are not part of the new O VI data set (Barstow et al. 2009). Finally, for completeness, O VI detections and non-detections towards B-type stars with distances $<120$ pc are also included on the plot (Bowen et al. 2008; Welsh and Lallement 2008).

It is clear from Fig. 4 that there is a remarkable spatial co-incidence between detections of local O VI absorption $(d<80 \mathrm{pc})$ and the highest levels of soft X-ray background emission. This is to be expected if $\mathrm{O}$ VI absorption is formed in a transition region between a hot $\left(T \sim 10^{6} \mathrm{~K}\right)$ and cooler $(T \sim 20,000 \mathrm{~K})$ plasma. The spatial distribution of $\mathrm{O}$ VI absorption shown in Fig. 4 can be explained by our presently proposed model if local O VI absorption $(d<100 \mathrm{pc})$ only occurs in only two environments: (i) at the interfaces between the Local Cavity and the overlying galactic halo (at the 'top' and 'bottom' of the Local Chimney) and (ii) at the neutral walls between the Local Cavity and nearby hot bubbles. The former distribution of O VI absorption is confirmed by the new O VI results in which $\mathrm{O}$ VI is only detected at high latitudes for sources with dis- non-detections are filled white circles or squares. Note that there are no detections of interstellar $\mathrm{O}$ VI along sight-lines $<80 \mathrm{pc}$ that are located near to the galactic plane. Also note that the $\mathrm{O}$ VI detections are well correlated spatially with the regions of highest soft X-ray background emission intensity at highly positive or negative galactic latitudes. The figure has been adapted from Barstow et al. (2009)

tance $>60$ pc (Barstow et al. 2008, 2009). The distribution of $\mathrm{O}$ VI at low latitudes is consistent with its formation at the interfaces between distant $(d>80 \mathrm{pc})$ bubble cavities and their surrounding neutral gas shells (Bowen et al. 2008; Welsh and Lallement 2008).

We conjecture that the above model can also explain many of the problems associated with the widely held hot gas model of the Local Hot Bubble listed in Sect. 2. In particular, this new model provides the following explanations for many of the problems outlined previously.

\subsection{The local cavity pressure problem}

There are now only two types of gas contained within the confines of the Local Cavity, i.e. a strongly photo-ionized ionized 'filler gas' at $T \sim 20,000 \mathrm{~K}$ and numerous partially ionized cloudlets of $T \sim 7000 \mathrm{~K}$, both of which may be in approximate pressure equilibrium. The million ${ }^{m o} \mathrm{~K}$, soft $\mathrm{X}$-ray emitting gas is now confined to high latitude sightlines at distances of at least 50-100 pc from the Sun. The hot, rarefied gas in the halo has a lower mass density than that in the disk, but it has a greater thermal pressure of $\sim 7$ to $10 \times 10^{3} \mathrm{~K} \mathrm{~cm}^{-3}$ for O VI-rich gas (Shelton et al. 2007). Ironically, in re-conceptualizing the local region, the thermal pressure has gone from being larger than that of the hot halo gas to being smaller. However, thermal pressures on the order of $\sim 3 \times 10^{3} \mathrm{~K} \mathrm{~cm}^{-3}$ are common throughout the disk, making the discrepancy between disk and halo pressure a widespread problem. Also, it is commonly assumed that other forms of pressure (including magnetic, cosmic ray and turbulent pressure) can make significant contributions to the total pressure of the ISM. The magnetic field 
strength of the neutral wall surrounding much of the Local Cavity in the disk (estimated at $\sim 8 \mu \mathrm{G}$, Andersson and Potter (2006), resulting in a magnetic pressure of $B^{2} / 8 \pi k$ of $\sim 18,000 \mathrm{~K} \mathrm{~cm}^{-3}$ ) demonstrates that it is possible for large magnetic pressures to exist. A magnetic field of this strength is very similar to that theorized for magnetic flux tubes that were originally invoked to constrain the number of production sites for interstellar O VI in the local ISM (Cox and Helenius 2003).

In addition, we note that global dynamic pressure equilibrium may not actually exist within the Local Cavity for several reasons. Firstly, the cavity is not fully pressure constrained by surrounding neutral interstellar gas walls and also there is clear evidence for the inflow of gas from both the adjacent Sco-Cen/Loop I cavity and from the lower galactic halo. Interestingly, it has been found that positive velocity $\mathrm{O}$ VI is mostly found at high latitudes in the northern galactic hemisphere, which has been interpreted as possibly being caused by an evaporative flow from a young interface between warm gas and a hot exterior (halo) medium (Savage and Lehner 2006). This scenario is similar to our Hot Top model in which the evaporative outflows of O VI are formed at the interface between the warm photo-ionized filler gas of the Local Cavity and that of the hotter infalling exterior gas of the inner halo located at the two openings of the Local Chimney.

\subsection{Size and shape of the local cavity}

In the Hot Top Model, the size and shape of the rarefied Local Cavity are simply defined by the neutral gas walls (as traced by $\mathrm{Na}$ I absorption). In previous explanations for the Local Hot Bubble, either the hot gas was assumed to fill the Local Cavity and thus take on its size and shape, or to fill a smaller region within the cavity such that the bubble's radius in any given direction scaled with the observed local X-ray intensity. The Hot Top Model easily resolves the difficulty with the first version of the Local Hot Bubble model and its inability to explain why, if the Local Cavity were filled with hot gas, the $\beta$ CMa tunnel direction wasn't found be brighter in soft X-ray emission than neighboring directions. This is no longer problematic if the tunnel is instead filled with lower temperature photo/collisionally ionized gas, as verified by several UV absorption studies of this region (Dupin and Gry 1998; Gry and Jenkins 2001). In addition, our model removes the somewhat contrived version of the Local Hot Bubble model which required a gap between the soft X-ray emitting hot gas and the neutral gas walls of the Local Cavity.

\subsection{Origin and age}

We note that interstellar structures similar to the Local Cavity and the Local Chimney have been detected elsewhere in both our own and other galaxies (McClure-Griffiths et al. 2003). Therefore, the presence of such a low-density cavity in the ISM that is linked to an overlying galactic halo is clearly not a rarity. Since our new model does not require the presence of significant amounts of local million degree $\mathrm{K}$ gas, then this suggests that either the Local Cavity is an old (fossil) SNR ( $T>10^{6}$ years) in which any hot gas has long since cooled and/or recombined, or that the Local Cavity was formed by some other manner, perhaps that which created Gould's Belt (Lallement 2008).

The origin and evolution of local blow-outs from the galactic disk have been detailed using hydrodynamic simulations (Breitschwerdt and de Avillez 2006; de Avillez and Breitschwerdt 2007). If the pressure of overlying halo gas is lower than the mean pressure in the disk, then interstellar bubbles (caused by the action of either stellar winds or supernova events) could blow out of the disk in the polar direction. Such structures would cool rapidly in the equatorial regions and O VI could arise in the upward traveling shock, and in the interfaces of the shocked cloudlets being ejected into the halo. We note that the numerous neutral and partially ionized gas clouds detected at both ends of the Local Chimney have mostly negative velocities, whereas the $\mathrm{O}$ VI bearing gas has mostly positive velocities (Savage and Lehner 2006). Thus, the Hot Top Model is really an observational description of an aging SNR whose hot gas has escaped and it is now filled with warm and photo-ionized gas, which explains the observed local dearth of H I. This warm gas may have entered the aging cavity through the adjacent Loop I interface, having being expelled as stellar winds from the Sco-Cen OB association.

\subsection{Kinematics of the local cavity gas}

The motions of both ionized and partially ionized gas within the Local Cavity (as measured by UV-visible absorption lines towards nearby stars) is governed by inflow from the adjacent interstellar bubbles that abut the Local Cavity (i.e. Loop I, Loop III, Pleiades etc.). No expansion is required (due to any pressure imbalance). The inflow of gas from the inner halo is seen only at $|z|>100 \mathrm{pc}$ and the lack of any observed general inflow from this infalling gas into the very inner regions of Local Cavity is most probably due to such an effect being masked by the gas flow vectors from the adjacent interstellar cavities.

\subsection{Absorption column densities}

In order for $\mathrm{O}$ VI absorption to occur, an interface between hot and cooler gas is required. In the new model this can occur at locations within the Local Chimney where infalling highly ionized million degree halo gas meets the cooler photoionized filler gas of the Local Cavity. The 
new O VI results support this scenario for sight-line distances $<100$ pc (Barstow et al. 2008, 2009). For sightlines in the galactic disk that extend beyond the confines of the Local Cavity, O VI absorption can occur at interfaces between more distant hot bubble cavities and their surrounding neutral gas shells (Welsh and Lallement 2008; Bowen et al. 2008). Clearly more distant $(d>200 \mathrm{pc})$ O VI absorption can also occur in more distant halo regions, as well as in distant hot superbubble cavities throughout the galactic plane.

\subsection{Soft X-ray background and EUV emission \& remaining problems}

Clearly the most radical proposition in our new model is the lack of a requirement for a ubiquitous million degree soft X-ray emitting gas contained within the confines of the Local Cavity, as delineated by its neutral absorbing walls. Although the contribution of solar wind charge exchange generated emission may account for a large fraction of the observed soft X-ray background (especially at low galactic latitudes), some of the models still require some contribution from a million degree gas present at low latitudes. This contribution is thought to be small $\left(<200 \times 10^{-6}\right.$ ROSAT counts s ${ }^{-1} \operatorname{arcmin}^{-2}$ ) and we propose that if it exists then its intrinsic variation in intensity with (galactic) sightline sampled has previously been masked by the far larger contribution to the total soft X-ray background signal generated by the foreground solar wind charge exchange emission. Therefore, if such hot gas does exist, then at present we know very little about its properties and previous models of the Local Hot Bubble require substantial revisions.

Although the new model attempts to provide explanations for many of the observational characteristics of soft $\mathrm{X}$-ray, EUV, UV and visible observations of the local interstellar gas, there is one set of observations that cannot be accounted for. From sounding rocket data gained over 25 years ago numerous observers have found that the $\mathrm{B} / \mathrm{Be}$ band ratio is essentially constant, irrespective of the sight-line probed. However, present calculations of the solar wind charge exchange emission at these very low energies suggests that very little emission arises within the B-band. We note that satellite observations of the diffuse EUV background (Jelinsky et al. 1995) require limits for the plasma emission measure of a factor of 5-10 below the B-band emission measured over a temperature range from $10^{5.7}-10^{6.4} \mathrm{~K}$. Also, the Be-band measurements by Cash et al. (1976), in agreement with the EUV satellite data, place a strict temperature upper limit of $\sim 3 \times 10^{5} \mathrm{~K}$ to any emission from a local plasma. Such conclusions are in direct conflict with other measurements gained at these soft energies (Bloch et al. 1986; Juda and Bloch 1991). Thus, the origin and physical nature of this near-isotropic flux, in the context of our new model, still remains to be found. However, we note that it must be due to a local hot gas, but with a lower temperature than 1 million ${ }^{m o} \mathrm{~K}$. One intriguing possibility is that such emission could arise in the terrestrial magnetosheath via charge exchange processes between heavy solar wind ions and geocoronal neutrals (Robertson and Cravens 2002).

Clearly, new high spectral resolution observations of the background emission in the 0.1 to $0.25 \mathrm{keV}$ band will be required in order to solve this outstanding issue. Such observations are non-trivial since they will require knowledge of the separate contributions to the total emission signal from solar wind charge exchange and a presumed more distant emitting hot gas.

\section{Conclusion}

The interstellar region surrounding the Sun to a distance of $\sim 80 \mathrm{pc}$ in all directions has historically been termed the Local Hot Bubble. Many of the physical properties of this gas, as revealed by observations of the diffuse soft X-ray background (soft X-ray background) emission, have long been described by emission from a million degree gas in thermal equilibrium. In this paper we have presented a wide variety of observational evidence gained over the last decade that would appear to contradict the expectations of a hot Local Bubble model. In particular, the recently realized nonnegligible contribution to the soft X-ray background signal from solar wind charge exchange $\mathrm{X}$-rays generated within the heliosphere has required a critical re-thinking of the various physical components that constitute the soft X-ray background spectrum.

We have provided arguments that would imply that, at the very least, the intensity of the soft X-ray background emission arising solely from a local million degree hot plasma must be reduced by $\sim 50 \%$. We have outlined the ramifications of such a reduction in the X-ray brightness of a Local Hot Bubble and, although several observations that have seemed at odds with the notion of an emitting Local Hot Bubble are now accounted for, the heating mechanism for the hot plasma must be critically re-evaluated now that the gas is found to be dimmer and cooler. In addition, the spatial distribution of the hot million degree gas within the Local Cavity also requires reconsideration.

Finally we present a new model of the Local Cavity (i.e. the 'Hot Top' model) in which solar wind charge exchange can explain all of the observed soft X-rays in the Galactic Plane, and thus the local interstellar medium is devoid of million degree plasma at mid-plane latitudes. Instead, the Local Cavity is filled with a very diffuse and photoionized and/or collisionally ionized gas of temperature $\sim 20,000 \mathrm{~K}$ that is in rough pressure equilibrium $\left(P / k \sim 3000 \mathrm{~cm}^{-3}\right)$ with the numerous partially ionized and warm $(T \sim 7000 \mathrm{~K})$ 
small cloudlets that are present within the local ISM. However, our new model does require the presence of a million degree emitting gas at high galactic latitudes, such that the associated soft X-ray emission is only detectable when viewed along sight-lines that extend beyond both ends of the Local Chimney feature into the lower galactic halo. The model also predicts that locally, the $\mathrm{O} \mathrm{VI}$ ion should be preferentially formed in interface regions between warm photoionized gas and the hot million degree gas of the halo. This scenario seems to be the case for O VI absorbers observed over sight-lines $<80 \mathrm{pc}$ from the Sun. The new model also provides an explanation for several observed parameters such as local gas pressure, the observed 'dipole' effect in maps of the soft X-ray background and the spatial concordance between the highest levels of soft X-ray emission and the spatial distribution of local O VI absorbers.

Although the Hot Top model can explain many observational conundrums, it presently provides no explanation for observations of the emission observed by sounding rockets in the B and Be-band X-rays. Such data are also in contradiction with observations of the EUV diffuse background emission recorded by satellite instrumentation. Future high resolution spectral observations with an X-ray microcalorimeter at ultra-soft X-ray wavelengths could provide important constraints on the (still) disputed physical nature of interstellar gas within $100 \mathrm{pc}$ of the Sun.

Acknowledgements BYW acknowledges financial support from NASA grant \# NNX07AE33G. RLS acknowledges financial support from NASA grant \# NNH072DA001N. Both authors would like to thank Don Cox, Steve Snowden, John Vallerga, Kip Kuntz, Martin Barstow, Ed Jenkins and Rosine Lallement for many interesting discussions concerning this topic, most of which took place while musing over a mug of fermented hops. We would also like to thank both the editor and referee for their help in improving this paper.

Open Access This article is distributed under the terms of the Creative Commons Attribution Noncommercial License which permits any noncommercial use, distribution, and reproduction in any medium, provided the original author(s) and source are credited.

\section{References}

Andersson, B.-G., Potter, S.: Astrophys. J. 640, L51 (2006)

Barstow, M., Boyce, D., Barstow, J., Forbes, A., Welsh, B.Y., Lallement, R.: Astrophys. Space Sci. 329, 91-95 (2008)

Barstow, M., Boyce, D., Welsh, B.Y. et al.: Astrophys. J. (2009, submitted)

Berghofer, T., Bowyer, S., Lieu, R., Knude, J.: Astrophys. J. 500, 838 (1998)

Bloch, J., Jahoda, K., Juda, M., et al.: Astrophys. J. 308, L59 (1986)

Bochkarev, N.: Sov. Astron. 31, 20B (1987)

Bohlin, R.: Astrophys. J. 200, 402 (1975)

Borkowski, K., Balbus, S., Fristrom, C.: Astrophys. J. 355, 501 (1990)

Bowen, D., Jenkins, E., Tripp, T., et al.: Astrophys. J. Suppl. Ser. 176, 59 (2008)

Bowyer, S., Field, G., Mack, J.: Nature 217, 32 (1968)

Breitschwerdt, D.: Astrophys. Space Sci. 276, 163 (2001)
Breitschwerdt, D., de Avillez, M.: Astron. Astrophys. 452, L1 (2006)

Breitschwerdt, D., Freyberg, M., Trumper, J.: In: IAU Colloq. 166, "The local bubble and beyond". LNP, vol. 506. Springer, Berlin (1998)

Breitschwerdt, D., Schmutzler, T.: Astron. Astrophys. 347, 650 (1999)

Bruhweiler, F., Cheng, K.-P.: Astrophys. J. 335, 188 (1988)

Burrows, D., Guo, Z.: In: IAU Colloq. 166, "The Local Bubble and Beyond". LNP, vol. 506, p. 279. Springer, Berlin (1998)

Burrows, D., Mendenhall, J.: Nature 351, 629 (1991)

Cash, W., Charles, P., Bowyer, S., et al.: Astrophys. J. 238, L71 (1980)

Cash, W., Malina, R., Stern, R.: Astrophys. J. 204, L7 (1976)

Chassefiere, E., et al.: Astron. Astrophys. 160, 229 (1986)

Chauvin, G., Menard, F., Fusco, T., et al.: Astron. Astrophys. 394, 949 (2002)

Comeron, F., Torra, J.: Astron. Astrophys. 281, 35 (1994)

Cox, D.: In: IAU Colloq. 166, "The Local Bubble and Beyond". LNP, vol. 506, p. 121. Springer, Berlin (1998)

Cox, D., Anderson, P.: Astrophys. J. 253, 268 (1982)

Cox, D., Helenius, L.: Astrophys. J. 583, 205 (2003)

Cox, D., Reynolds, R.: Annu. Rev. Astron. Astrophys. 25, 303 (1987)

Cox, D., Smith, B.: Astrophys. J. 189, L105 (1974)

Cravens, T.: Geophys. Res. Lett. 24, 105 (1997)

Cravens, T.: Astrophys. J. 532, L153 (2000)

Cravens, T., Robertson, I., Snowden, S.: J. Geophys. Res. 106, 24883 (2001)

Crawford, I., Lallement, R., Price, R., et al.: Mon. Not. R. Astron. Soc. 337, 720 (2002)

Crawford, I., Lallement, R., Welsh, B.Y.: Mon. Not. R. Astron. Soc. 300, 1181 (1998)

de Avillez, M., Breitschwerdt, D.: Astrophys. J. 665, L35 (2007)

Dupin, O., Gry, C.: Astron. Astrophys. 335, 661 (1998)

Edelstein, J., Bowyer, S., Korpela, E., et al.: Astrophys. Space Sci. 276, $177(2001)$

Edgar, R., Cox, D.: Astrophys. J. 413, 190 (1993)

Ferlet, R., Vidal-Madjar, A., Gry, C.: Astrophys. J. 298, 838 (1985)

Fields, B., Hochmuth, K., Ellis, J.: Astrophys. J. 621, 902 (2005)

Fitzgerald, M.: Astron. J. 73, 983 (1968)

Freyberg, M.: In: IAU Colloq. 166, "The Local Bubble and Beyond". LNP, vol. 506, p. 113. Springer, Berlin (1998)

Frisch, P.: Nature 293, 377 (1981)

Frisch, P.: Space Sci. Rev. 72, 499 (1995)

Frisch, P., York, D.: Astrophys. J. 271, L59 (1983)

Fuchs, B., Breitschwerdt, D., de Avillez, M., et al.: Mon. Not. R. Astron. Soc. 373, 993 (2006)

Galleazzi, M., Gupta, A., Covey, K., Ursino, E.: Astrophys. J. 658, 1081 (2007)

Grenier, I.: Astron. Astrophys. 364, L93 (2000)

Gry, C., Jenkins, E.: Astron. Astrophys. 367, 617 (2001)

Heiles, C.: Astrophys. J. 229, 533 (1979)

Henley, D., Shelton, R., Kuntz, K.: Astrophys. J. 661, 304 (2007)

Holberg, J., Bruhweiler, F., Barstow, M., Dobbie, P.: Astrophys. J. 517, 841 (1999)

Hurwitz, M., Sasseen, T., Sirk, M.: Astrophys. J. 623, 911 (2005)

Jelinsky, P., Vallerga, J., Edelstein, J.: Astrophys. J. 442, 653 (1995)

Jenkins, E.: Astrophys. J. 219, 845 (1978)

Jenkins, E.: Astrophys. J. 580, 939 (2002)

Jenkins, E.: Space Sci. Rev. 143, 205-216 (2008)

Jenkins, E., Meloy, D.: Astrophys. J. 193, L121 (1974)

Juda, M., Bloch, J., Edwards, B., et al.: Astrophys. J. 367, 182 (1991)

Knie, K., et al.: Phys. Rev. Lett. 93, 1103 (2004)

Koutroumpa, D., Acero, F., Lallement, R., et al.: Astron. Astrophys. 475, 901 (2007)

Koutroumpa, D., Lallement, R., Kharchenko, V., et al.: Astron. Astrophys. 460, 289 (2006)

Koutroumpa, D., Lallement, R., Kharchenko, V., et al.: Space Sci. Rev. 143, 217-230 (2008a) 
Koutroumpa, D., Lallement, R., Raymond, J.C., Kharchenko, V.: Astron. Astrophys. (2009, submitted)

Kuntz, K., Snowden, S.: Astrophys. J. 543, 195 (2000)

Kuntz, K., Snowden, S., Verter, F.: Astrophys. J. 484, 245 (1997)

Lallement, R.: Astron. Astrophys. 418, 143 (2004)

Lallement, R.: Space Sci. Rev. 143, 427-436 (2008)

Lallement, R., Bertin, P.: Astron. Astrophys. 266, 479 (1992)

Lallement, R., Vidal-Madjar, A., Ferlet, R.: Astron. Astrophys. 168, 225 (1986)

Lallement, R., Welsh, B.Y., Vergely, J.L., Crifo, F., Sfeir, D.: Astron. Astrophys. 411, 447 (2003)

Lehner, N., Jenkins, E., Gry, C., et al.: Astrophys. J. 595, 858 (2003)

Lucke, P.: Astron. Astrophys. 64, 367 (1978)

Luhman, K.: Astrophys. J. 560, 287 (2001)

Lyu, C.-H., Bruhweiler, F.: Astrophys. J. 459, 216 (1996)

Maiz-Apellaniz, J.: Astrophys. J. 560, L83 (2001)

Markevitch, M., Bautz, M., Biller, B., et al.: Astrophys. J. 583, 70 (2003)

Mathewson, D., Ford, V.: Mon. Not. R. Astron. Soc. 74, 139 (1970)

McCammon, D., Sanders, W.: Annu. Rev. Astron. Astrophys. 28, 657 (1990)

McCammon, D., Meyer, S., Sanders, W., Williamson, F.: Astrophys. J. 209, 46 (1976)

McCammon, D., Almy, R., Apodaca, E., et al.: Astrophys. J. 576, 188 (2002)

McClure-Griffiths, N., Dickey, J., Gaensler, B., Green, A.: Astrophys. J. 594, 833 (2003)

McKee, C., Ostriker, J.: Astrophys. J. 218, 148 (1978)

Nehme, C., Gry, C., Boulanger, F., et al.: Astron. Astrophys. 483, 471 (2008)

Oegerle, W., Jenkins, E., Shelton, R., et al.: Astrophys. J. 622, 377 (2005)

Redfield, S., Linsky, J.: Astrophys. J. 673, 283 (2008)

Reynolds, R., Ogden, P.: Astrophys. J. 229, 942 (1979)

Robertson, I., Cravens, T.: J. Geophys. Res. 108(A10), 8031 (2003)

Robertson, I., Cravens, T.: Geophys. Res. Lett. 30, 1439 (2002)

Sanders, W., Edgar, R., Kraushaar, W., McCammon, D., Morgenthaler, J.: Astrophys. J. 554, 694 (2001)

Sanders, W., Kraushaar, W., Nousek, J., et al.: Astrophys. J. 217, L87 (1977)

Savage, B., Lehner, N.: Astrophys. J. Suppl. Ser. 162, 134 (2006)

Sfeir, D., Lallement, R., Crifo, F., Welsh, B.Y.: Astron. Astrophys. 346, 785 (1999)

Shelton, R.: Astrophys. J. 504, 785 (1998)

Shelton, R.: Astrophys. J. 589, 261 (2003)

Shelton, R., Sallmen, S., Jenkins, E.: Astrophys. J. 659, 365 (2007)
Slavin, J.: Astrophys. J. 346, 718 (1989)

Slavin, J., Frisch, P.: Astrophys. J. 565, 364 (2002)

Smith, R., Bautz, M., Edgar, R., et al.: Publ. Astron. Soc. Pac. 59, S141 (2007)

Smith, R.K., Cox, D.: Astrophys. J. Suppl. Ser. 134, 283 (2001)

Smith, R., Edgar, R., Plucinski, P., et al.: Astrophys. J. 623, 225 (2005)

Snowden, S., Cox, D., McCammon, D., Sanders, W.: Astrophys. J. 354, $211(1990)$

Snowden, S., McCammon, D., Verter, F.: Astrophys. J. 409, L21 (1993)

Snowden, S., Egger, R., Freyberg, M., et al.: Astrophys. J. 485, 125 (1997)

Snowden, S., Egger, R., Finkbeiner, D., et al.: Astrophys. J. 493, 715 (1998)

Snowden, S., Freyberg, M., Kuntz, K., Sanders, W.: Astrophys. J. Suppl. Ser. 128, 171 (2000)

Sofia, U., Jenkins, E.: Astrophys. J. 499, 951 (1998)

Tinbergen, J.: Astron. Astrophys. 105, 53 (1982)

Vallerga, J.: Astrophys. J. 497, 921 (1998)

Vallerga, J., Slavin, J.: In: IAU Colloq. 166, "The Local Bubble and Beyond". LNP, vol. 506, p. 79. Springer, Berlin (1998)

Vallerga, J., Welsh, B.Y.: Astrophys. J. 444, 202 (1995)

Wargelin, B., Beiersdorfer, P., Brown, G.: Can. J. Phys. 86, 151 (2008)

Weaver, R., McCray, R., Castor, J., Shapiro, P., Moore, R.: Astrophys. J. 218, 377 (1977)

Welsh, B.Y., Lallement, R.: Astron. Astrophys. 436, 615 (2005)

Welsh, B.Y., Lallement, R.: Astron. Astrophys. 490, 707 (2008)

Welsh, B.Y., Craig, N., Vedder, P., Vallerga, J.: Astrophys. J. 437, 638 (1994)

Welsh, B.Y., Crifo, F., Lallement, R.: Astron. Astrophys. 333, 10 (1998)

Welsh, B.Y., Sfeir, D., Sirk, M., Lallement, R.: Astron. Astrophys. 352, 308 (1999)

Welsh, B.Y., Sallmen, S., Sfeir, D., et al.: Astron. Astrophys. 394, 691 (2002)

Welsh, B.Y., Sallmen, S., Lallement, R.: Astron. Astrophys. 414, 261 (2004)

Williamson, F., Sanders, W., Kraushaar, W., et al.: Astrophys. J. 193, L133 (1974)

Wolff, B., Koester, D., Lallement, R.: Astron. Astrophys. 346, 969 (1999)

Wolleben, M.: Astrophys. J. 664, 349 (2007)

Wood, B., Izmodenov, V., Linsky, J., Malama, Y.: Astrophys. J. 657, 609 (2007)

Yao, Y., Schulz, N., Wang, D., et al.: Astrophys. J. 653, L121 (2006) 